\title{
The Use of Thymalin for Immunocorrection and Molecular Aspects of Biological Activity
}

\author{
V. Kh. Khavinson ${ }^{a, b}$, N. S. Linkova ${ }^{a, c, d, *}$, N. I. Chalisova ${ }^{a, b}$, and O. M. Ivko ${ }^{a}$ \\ ${ }^{a}$ St. Petersburg Institute of Bioregulation and Gerontology, St. Petersburg, Russia \\ ${ }^{b}$ Pavlov Institute of Physiology, Russian Academy of Sciences, St. Petersburg, Russia \\ ${ }^{c}$ Academy of Postgraduate Education of the Federal State Budgetary Institution, the Federal Science and Clinic Center \\ of the Federal Medical and Biological Agency, Moscow, Russia \\ ${ }^{d}$ Belgorod State University, Belgorod, Russia \\ *e-mail:miayy@yandex.ru \\ Received June 12, 2020; revised June 12, 2020; accepted June 12, 2020
}

\begin{abstract}
The polypeptide drug thymalin is used for various diseases associated with immune dysfunction, viral and bacterial infections, regeneration normalization, immunodepression, and the depression of hematogenesis after chemical and radiotherapy. The molecular mechanism of the action of thymalin and its components, EW dipeptide (the drug thymogen), the dipeptide KE, and the tripeptide EDP, are analyzed. These short peptides regulate gene expression and the synthesis of heat-shock protein, cytokines, fibrinolysis, gerontogenes, and the differentiation, proliferation, apoptosis of cells. Thymalin and thymogen have practically no side effects and are used for various viral infections. These peptide drugs can likely be effective in the complex therapy for the coronavirus infection COVID-19.
\end{abstract}

Keywords: thymalin, thymogen, short peptides, mechanisms of action, immunocorrection

DOI: $10.1134 / \mathrm{S} 2079086421040046$

The creation of effective and safe drugs that can increase the protective functions of the body is a highpriority area of molecular medicine and immunology. The peptides formed via limited proteolysis are endocrine and autocrine carriers of information about the local state of the functions of an organ or tissue. Low molecular weight short peptides containing up to ten amino acid residues have a wide spectrum of activity and coordinate the biological functions of various organs and tissues (Ryzhak et al., 2015; Khavinson and Popovich, 2017). There are a large number of peptides that are known that regulate the differentiation, proliferation, and apoptosis of cells in normal conditions with pathology and aging of the body (Khavinson et al., 2020).

A technology has been developed for the isolation of polypeptide complexes from various organs and tissues of young animals. The first peptide drug created with this technology was a polypeptide complex isolated from the thymus of calves, thymalin (Morozov and Khavinson, 1981; Morozov et al., 1982; Khavinson and Morozov, 1982). This drug normalizes the functions of the immune system and stimulates the processes of regeneration and hematopoiesis if they are suppressed. The immunoprotective effect of thy- malin is expressed in the restoration of the number and ratio of $\mathrm{T}$ and $\mathrm{B}$ lymphocytes, their subpopulations, an increase in their functional activity, and increased phagocytosis (Morozov et al., 2000; Anisimov and Khavinson, 2010). Timalin is used in the treatment of various diseases associated with dysfunction of the immune system in children and adults (acute and chronic viral and bacterial infections, infectious purulent and septic processes, impaired regeneration processes, suppression of immunity and hematopoiesis after chemotherapy or radiation therapy) (Khavinson et al., 1991, 2013, 2014a; Khavinson and Morozov, 2002; Kuznik et al., 2002; Khlystova et al., 2003). The use of thymalin helped to reduce the incidence of acute respiratory diseases in elderly and senile people by 2.0-2.4 times (Khavinson and Morozov, 2002). It was found that thymalin accumulates in epithelial cells of the human respiratory tract during early embryogenesis (Khlystova et al., 2003). The use of thymalin in the treatment of patients with acute lung abscesses led to a decrease in the systemic inflammatory response syndrome, a decrease in plasma fibrinolytic activity, and the cessation of hypercoagulation (Kuznik et al., 2002). Timalin has shown geroprotective properties in animal experiments and in clinical studies. The drug contributed to the restoration of functions of the 
immune system and reduced mortality in elderly patients (Korkushko et al., 2002; Anisimov and Khavinson, 2010). Thus, thymalin normalizes the functions of the immune and respiratory systems, and, in addition, has a geroprotective effect. The use of thymalin in the experiment and in patients with pneumonia of various etiologies, viral infectious diseases was accompanied by the normalization of blood coagulation and fibrinolytic activity (Kuznik et al., 2011, 2013; Khavinson et al., 2012b, 2013).

Immunomodulatory therapy and drugs that normalize the hemostasis system are currently a promising approach to the complex treatment of the viral disease COVID-19, the severe form of which is accompanied by pneumonia, thrombus formation, and an imbalance in the cytokine status. Given that the risk group for COVID-19 is mainly elderly and senile people, thymalin can be considered a drug for the complex therapy of infection caused by SARS-CoV-2 (severe acute respiratory syndrome coronavirus 2) (Tang et al., 2020; Wang et al., 2020; Zhang et al., 2020).

Thus, it is important to study the molecular aspects of the mechanism of action of thymalin. It should be noted that the active components of a polypeptide preparation contain short peptides (Khavinson et al., 2017). Earlier, the dipeptide EW was isolated from thymalin via reversed-phase high-performance liquid chromatography (RP-HPLC); it was later synthesized and named "thymogen." Further experimental and clinical studies have shown that thymogen is a highly effective immunomodulator (Morozov et al., 2000). The short peptides in thymalin were identified via mass spectrometry and HPLC (Zhurkovich et al., 2020). The dipeptide KE (vilon) and the tripeptide EDP (crystagen), which have high biological activity, were identified in the study.

The areas of the application of the drugs thymalin and thymogen are in many ways similar. The use of thymogen is indicated for the prevention and complex treatment of acute and chronic viral and bacterial diseases of the upper respiratory tract and the prevention of the suppression of immunity, hematopoiesis, and regeneration processes in the posttraumatic and postoperative periods. A double-blind, randomized, placebo-controlled study of the efficacy of thymogen in elderly patients after the removal of solid tumors in the abdominal cavity and retroperitoneal space was carried out. The preoperative use of thymogen restored the structural and functional parameters of immunity and led to a statistically significant decrease in the number and range of postoperative complications and a reduction in the postoperative period (Smirnov et al., 2011). Thymogen, like thymalin, demonstrated geroprotective properties in animal experiments. Like thymalin, the use of thymogen in experiment and in patients with pneumonia of various etiologies and viral infectious diseases was accompanied by normalization of the coagulation and fibrinolytic activity of blood plasma proteins (Kuznik et al., 2011; Khavinson et al., 2013). It should be noted that thymogen administration in rats led to an increase in survival and a 1.5-fold decrease in the number of tumors (Anisimov et al., 2000).

It was found that the short peptides thymogen, vilon, and crystagen activate the differentiation, increase the viability and proliferation, and reduce the apoptosis of various subpopulations of immune cells.

It was found that the use of the KE peptide stimulates cellular immunity and nonspecific resistance of the organism and has an activating effect on macrophages, blood lymphocytes, thymocytes, and neutrophils (Shcherbak et al., 2004; Sevostyanova et al., 2012). Vilon induces the expression of CD4 and CD5 molecules on thymus cells, stimulating their differentiation in the direction of $\mathrm{T}$ helpers (Sevostyanova et al., 2012). The KE peptide, when introduced into the body of transgenic mice, suppresses the expression of the oncogene $H E R-2 /$ neu by two times (Khavinson, 2009 ). Vilon promotes an increase in the proportion of transcribed euchromatin and a decrease in the amount of heterochromatin in the blood lymphocytes of elderly people (Khavinson et al., 2004). The KE peptide normalizes the telomere length of PHA-stimulated blood lymphocytes in people of different ages (Khavinson et al., 2019). Vilon also regulates the gene expression of IGF1, FOXO1, TERT, TNKS2, and $N F K B$ in human embryonic mesenchymal stem cells of the FetMSC line during their replicative and stationary aging (Ashapkin et al., 2020). The KE peptide was found to be one of the 57 dipeptides capable of selective binding to double-stranded DNA. When studying the effect of peptides on the expression of 15247 genes with microchip technology, it was found that the KE peptide regulates the gene expression of EPS15, MCM10 homolog, Culline 5, APG5L, FUSED, ZNF01, FLJ12848 fis, ITPK1, SLC7A6, FLJ22439 fis, KIAA0029, FLJ13697 fis, KIAA0699, FLJ10914, Gdap1, MSTP028, MLLT3, and PEP, as well as the synthesis of cytoskeleton proteins and the proliferation and metabolism of cells, which explains its high biological activity (Khavinson and Malinin, 2005). It was found in the experiment the KE peptide demonstrates pronounced selective binding to the TCGA DNA sequence, which is part of these genes. It is assumed that the KE peptide can regulate gene expression and the synthesis of heat-shock proteins (HSPs), cytokines (IL-3, IL-4, IL-5, IL-6, IL-10, IL-17A, $\mathrm{TNF} \alpha$ ), tissue factor, proteins of the fibrinolysis system (plasminogen, tissue and urokinase plasminogen activators, antithrombin III), and proteins of "youth" 
and "old age" (GDF11, FNDC5, betatrophin, FGF19, FGF21, FGF23, HMGB1, MIC1/GDF15, JAM-A) (Khavinson et al., 2011a, 2014b, 2015a, 2015b, 2018; Kuznik et al., 2014, 2017). It was found that the KE motif is found in the amino-acid sequences of some cytokines and peptide hormones that are similar in function to the KE peptide. In the human proteome, nuclear proteins contain the largest number of KE motifs, while cytoplasmic and all other proteins have the minimal KE content. During limited proteolysis, the KE-peptide molecules released from nuclear proteins can bind to DNA and regulate gene expression (Terekhov et al., 2019). The KE peptide reduces the expression of matrix metalloproteinase-9 (MMP-9) and increases the expression of Ki-67 and CD98hc molecules, the synthesis of which is impaired with the aging of skin fibroblasts (Linkova et al., 2016).

The EDP peptide promotes heterochromatinization of the chromatin in blood lymphocytes in the elderly (Khavinson et al., 2006). The effectiveness of the oral administration of crystagen was evaluated in elderly and senile people with impaired immunity. Crystagen, in combination with standard treatment, contributed to the normalization of the immunogram in $82 \%$ of patients, while the normalization of the immunogram was observed in $56 \%$ of patients in the control group (standard treatment only). Crystagen had a greater effect on the T-cell element of immunity (an increase in the number of $\mathrm{CD}^{+}-, \mathrm{CD}^{+}$-cells, normalization of the $\mathrm{CD} 4^{+} / \mathrm{CD}^{+}$ratio) and had a less pronounced effect on $\mathrm{B}$ lymphocytes. In all patients taking crystagen, there was a decrease in the severity of asthenic syndrome, which accompanies secondary immunodeficiencies (Khavinson et al., 2006). The immunogeroprotective effect of crystagen on thymic cells of rats exposed to gamma irradiation (accelerated aging model) was established. After the introduction of crystagen, the animals retained the cortex-medulla division in the thymus (its absence is a sign of aging), and there were increases in the number of macrophages and mast cells and the proliferation of thymocytes, which was assessed based on the expression of proliferating cell nuclear antigen (PCNA) (Khavinson et al., 2006). The EDP peptide wash shown to have a stimulating effect on the ability of peritoneal macrophages of young and old mice to secrete the cytokines IL-1, IL-6, TNF $\alpha$. In addition, the EDP peptide activates the proliferation of VTEC2.H/S human thymic epithelial cells (Khavinson et al., 2006). The EDP peptide inhibits the proliferation of human erythromyelosis tumor K-562 cells. The inhibition of proliferation under the action of crystagen, which is directed at immortalized cells, may indicate its antitumor activity. The EDP peptide enhances the spontaneous proliferative activity of normal lymphocytes; this shows that it has a stimulating effect on nontumor human immune cells (Khavinson et al., 2011b). The oral administration of crystagen in combination with other short peptides increases stress resistance and normalizes immunity in athletes. Peptides doubled the expression of the HSP gene HSPA1A and normalized $I L-6$ expression. After the administration of peptide bioregulators, the incidence of acute respiratory viral infections in athletes decreased (Khavinson et al., 2012a).

Studies involving a comparative study of thymalin and the short peptides EW, KE, and EDP for immunogenesis were carried out. In organotypic cultures of spleen tissue of young and old rats, the effect of peptides on the processes of cell proliferation and apoptosis (lymphoblasts, lymphocytes, macrophages) was studied. Timalin and the peptides EW, KE, and EDP increased the index of the growth zone of the animal spleen explants by $20-50 \%$, stimulated proliferation, and reduced cell apoptosis by $29-42 \%$ as compared to the control. The effect of thymalin was more pronounced than that of short peptides (Zakutskii et al., 2006; Chalisova et al., 2008, 2011, 2015; Chervyakova et al., 2014; Ryzhak et al., 2017). The obtained data can be explained by the fact that thymalin contains all three studied short peptides, as well as a large number of other peptides that may have immunoprotective properties.

The effect of the EW, KE, and EDP peptides on the expression of markers of receptor (proteins p16, p21, p53, Caspase-8, -9) and mitochondrial (AIF, prohibitin) apoptosis in cultures of human blood lymphocytes was studied. It was found that these peptides reduce the level of receptor and mitochondrial apoptosis in blood lymphocytes during their replicative aging. The EW and KE peptides have a more pronounced antiapoptotic effect in "young" and "old" cultures of blood lymphocytes than the EDP peptide (Dudkov, 2019). It is assumed that the antiapoptotic effect of peptides may be associated not only with their ability to epigenetically regulate gene expression but also with another mechanism. The antiapoptotic and geroprotective effect of peptides can be realized via activation of the transcription of apoptosis genes when the conformation of histones changes under the influence of short peptides. It was established via molecular modeling that peptides bind most preferentially to histones $\mathrm{H} 1 / 1, \mathrm{H} 1 / 3, \mathrm{H} 1 / 6, \mathrm{H} 2 \mathrm{~b}, \mathrm{H} 3, \mathrm{H} 4$ at sites that interact with DNA. The most energetically favorable interaction with histone $\mathrm{H} 1 / 3$ occurs with the EW peptide.

The information entering a living organism is controlled by regulatory systems, which work to maintain a high degree of stability of genome functioning. Consequently, the main task of the bioregulation system is 


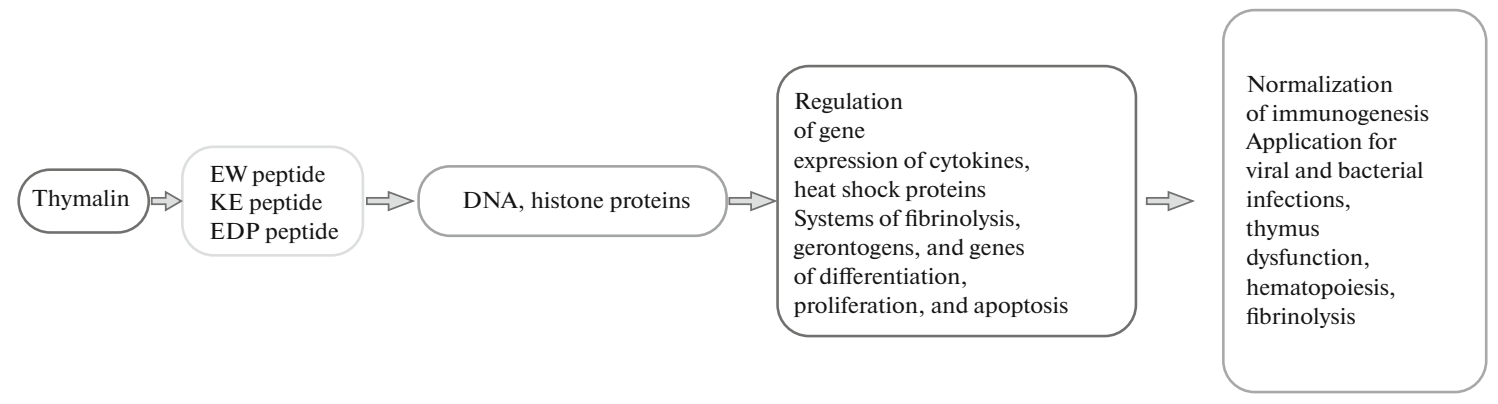

Fig. 1. Proposed scheme for the implementation of the molecular-biological effects of thymalin and its constituent short peptides.

to control gene expression, protein-synthesis processes, and protective functions of the body. Information on changes in the external and internal environment serves as a factor that induces transformation in the bioregulation system, which is necessary to maintain the functional activity of cells. Therefore, the interaction of information molecules, in particular, regulatory peptides, with the genome plays an important role in the maintenance of cellular homeostasis.

The immune and geroprotective effects of thymalin, which normalizes differentiation and proliferation and reduces apoptosis of lymphoid cells and activates cellular immunity reactions and regeneration processes, are due to the effects of its short peptides EW, $\mathrm{KE}$, and EDP. The EW, KE, and EDP peptides in the composition of the thymalin drug are able to complementarily bind to certain DNA sequences or histone proteins. As a result of this binding, there is a change in the gene expression and synthesis of HSPs, cytokines, the fibrinolysis system, gerontogens, and proteins involved in the differentiation, proliferation, and apoptosis of immune cells. Normalization of the synthesis of proteins that activate functions of the immune and antioxidant systems, the processes of regeneration, and hemostasis is manifested by restoration of the functions of the regulatory systems of the body and explains the high efficiency of thymalin in the treatment of a wide range of diseases (Fig. 1). Since thymalin and its constituent thymogen are used for viral infections and for bronchoprotection, it can be assumed that these drugs will be effective in the complex therapy of coronavirus infection. All of this indicates the relevance of further studies of thymalin and its constituent short peptides.

\section{COMPLIANCE WITH ETHICAL STANDARDS}

Conflict of interests. The authors declare that they have no conflicts of interest.

Statement on the welfare of humans or animals. All applicable international, national, and/or institutional guidelines for the care and use of animals were followed.

\section{REFERENCES}

Anisimov, V.N. and Khavinson, V.Kh., Peptide bioregulation of aging: results and prospects, Biogerontology, 2010, vol. 11, pp. 139-149. https://doi.org/10.1007/s10522-009-9249-8

Anisimov, V.N., Khavinson, V.Kh., and Morozov, V.G., Immunomodulatory synthetic dipeptide L-Glu-L-Trp slows down aging and inhibits spontaneous carcinogenesis in rats, Biogerontology, 2000, vol. 1, pp. 55-59. https://doi.org/10.1023/a:1010042008969

Ashapkin, V., Khavinson, V., Shilovsky, G., et al., Gene expression in human mesenchymal stem cell aging cultures: modulation by short peptides, Mol. Biol. Rep., 2020, vol. 47, pp. 4323-4329. https://doi.org/10.1007/s11033-020-05506-3

Chalisova, N.I., Lesnyak, V.V., Oganezova, E.V., and Rizhak, G.A., Protective effect of tripeptide in the presence of cyclophosphamide on the growth of cultured lymphoid tissue from rats of different age, Bull. Exp. Biol. Med., 2008, vol. 145, no. 6, pp. 748-750.

Chalisova, N.I., Linkova, N.S., Dudkov, A.V., and Kontsevaya, E.A., Effect of peptides on the proliferation and differentiation of spleen B-lymphocytes, Vestn. Belgorod. Gos. Univ., 2011, vol. 3, pp. 94-97.

Chalisova, N.I., Ryzhak, A.P., Lin'kova, N.S., et al., The effect of polypeptides on cell regeneration in a culture of different tissues of young and old rats, Usp. Gerontol., 2015, vol. 28, no. 1, pp. 93-107.

Ryzhak, A.P., Chalisova, N.I., Lin'kova, N.S., et al., Effect of polypeptides on cell proliferation and apoptosis during aging, Bull. Exp. Biol. Med., 2017, vol. 162, no. 4, pp. 534-538.

Chervyakova, N.A., Lin'kova, N.S., Chalisova, N.I., et al., Age-related molecular aspects of immunomodulating activity of peptides in the spleen, Adv. Gerontol., 2014, vol. 4 , no. 1 , pp. $12-15$.

Dudkov, A.V., Peptide regulation of caspase-dependent apoptosis during cellular senescence, Sovrem. Probl. Nauki Obraz., 2019, no. 2, pp. 1-11. http://www.science-education.ru/article/view?id=28701.

Khavinson, V.Kh., Peptidnaya regulyatsiya stareniya (Peptide Regulation of Aging), St. Petersburg: Nauka, 2009.

Khavinson, V.Kh. and Malinin, V.V., Gerontological Aspects of Genome Peptide Regulation, Basel: Karger, 2005.

Khavinson, V.Kh. and Morozov, V.G., Experimental and clinical study of thymalin-a new immunoregulatory drug, Voen.-Med. Zh., 1982, no. 5, pp. 37-39. 
Khavinson, V.Kh. and Morozov, V.G., Geroprotective efficiency of thymalin and epithalamin, Usp. Gerontol., 2002, no. 10, pp. 74-84.

Khavinson, V. and Popovich, I., Short peptides regulate gene expression, protein synthesis and enhance life span, in Anti-Aging Drugs: From Basic Research to Clinical Practice, RSC Drug Discovery Series no. 57, Vaiserman, A.M., Ed., Cambridge, 2017, ch. 20, pp. 496-513.

Khavinson, V.Kh., Seryi, S.V., and Malinin, V.V., Correction radiation disorders of immuno- and hematopoiesis by thymus and bone marrow peptides, Radiobiologiya, 1991, vol. 31, no. 4, pp. 501-505.

Khavinson, V.K., Lezhava, T.A., and Malinin, V.V., Effects of short peptides on lymphocyte chromatin in senile subjects, Bull. Exp. Biol. Med., 2004, vol. 137, no. 1, pp. 78-81.

Khavinson, V.Kh., Grigor'ev, E.I., Malinin, V.V., and Ryzhak, G.A., RF Patent 2301074, 2006.

Khavinson, V.Kh., Kuznik, B.I., and Linkova, N.S., Effect of peptide bioregulators and cytokines on life span and age-related changes of hemostasis, Tromboz, Gemostaz, Reol., 2011a, no. 4, pp. 15-32.

Khavinson, V.K., Nikolsky, I.S., Nikolskaya, V.V., et al., Effect of tripeptides on lymphoid and stem cells, Bull. Exp. Biol. Med., 2011b, vol. 151, no. 6, pp. 722-725.

Khavinson, V.Kh., Viner, I.A., Trofimova, S.V., et al., Metodika povysheniya rezervnykh vozmozhnostei organizma sportsmenov vysokoi kvalifikatsii, spetsializiruyushchikhsya v slozhnokoordinatsionnykh vidakh sporta, s pomoshch'yu peptidnykh bioregulyatorov. Metodicheskie rekomendatsii (A Method for Improvement of Reserves of Organisms of Sportsmen Specializing in Complex Sport Types Using Peptide Bioregulators: Methodological Recommendations), St. Petersburg: Inst. Bioregul. Gerontol., 2012.

Khavinson, V.K., Kuznik, B.I., and Ryzhak, G.A., Peptide bioregulators: a new class of geroprotectors. Message 1: Results of experimental studies, Adv. Gerontol., 2013, vol. 3, no. 3, pp. 225-235.

Khavinson, V.Kh., Kuznik, B.I., and Ryzhak, G.A., Peptidnye geroprotektory-epigeneticheskie regulyatory fiziologicheskikh funktsii organizma (Peptide GeroprotectorsEpigenetic Regulators of Physiological Functions of the Organism), St. Petersburg: Ross. Gos. Pedagog. Univ. im. A.I. Gertsena, 2014a.

Khavinson, V.Kh., Kuznik, B.I., and Ryzhak, G.A., Peptide bioregulators: a new class of geroprotectors, Report 2. The results of clinical trials, Adv. Gerontol., 2014b, vol. 4, no. 4, pp. 346-361.

Khavinson, V.Kh., Kuznik, B.I., Tarnovskaya, S.I., et al., Peptides and CCL11 and HMGB1 as molecular markers of aging: literature review and own data, Adv. Gerontol., 2015, vol. 5, no. 3, pp. 133-140.

Khavinson, V.Kh., Kuznik, B.I., Tarnovskaya, S.I., et al., GDF11 protein as a geroprotector, Biol. Bull. Rev., 2016a, vol. 6, no. 2, pp. 141-148.

Khavinson, V.Kh., Kuznik, B.I., Tarnovskaya, S.I., et al., Short peptides and telomere length regulator hormone irisin, Bull. Exp. Biol. Med., 2016b, vol. 160, no. 3, pp. 347-349.
Khavinson, V.Kh., Kopylov, A.T., Vaskovsky, B.V., et al., Identification of peptide AEDG in the polypeptide complex of the pineal gland, Bull. Exp. Biol. Med., 2017, vol. 164, no. 3 , pp. 41-43.

Khavinson, V.Kh., Kuznik, B.I., Ryzhak, G.A., et al., Irisin, betatrophin, diabetes mellitus, obesity, and metabolic syndrome: epigenetic mechanisms of regulation, Usp. Fiziol. Nauk, 2018, vol. 49, no. 1, pp. 72-86

Khavinson, V.Kh., Linkova, N.S., Pendina, A.A., et al., The effect of the KE peptide on the telomere length of the chromosomes of PHA-stimulated human lymphocytes, Med. Akad. Zh., 2019, special issue, pp. 166-168.

Khavinson, V., Linkova, N., Diatlova, A., and Trofimova, S., Peptide regulation of cell differentiation, Stem Cell Rev. Rep., 2020, vol. 16, pp. 118-125.

https://doi.org/10.1007/s12015-019-09938-8

Khlystova, Z.S., Kalinina, I.I., and Shmeleva, S.P., Thymalin in developing respiratory organs of human fetus, Bull. Exp. Biol. Med., 2003, vol. 135, pp. 600-602. https://doi.org/10.1023/a:1025449923475

Korkushko, O.V., Khavinson, V.Kh., Butenko, G.M., and Shatilo, V.B., Peptidnye preparaty timusa i epifiza $v$ profilaktike uskorennogo stareniya (Peptide Preparations of the Thymus and the Pineal Gland in the Prevention of Accelerated Aging), St. Petersburg: Nauka, 2002.

Kuznik, B.I., Budazhabon, G.B., Darenskaya, S.D., and Likhanov, I.D., The effect of thymalin on immunity and hemostasis in patients with lung abscesses, Tromboz, Gemostaz, Reol., 2002, no. 3 (11), pp. 55-61.

Kuznik, B.I., Linkova, N.S., and Khavinson, V.K., Heat shock proteins: changes related to aging, development of thrombotic complications, and peptide regulation of the genome, Adv. Gerontol., 2012, vol. 2, no. 3, pp. 175186.

Kuznik, B.I., Linkova, N.S., Tarnovskaya, S.I., and Khavinson, V.Kh., Cytokinis and regulatory peptides: age-related changes, atherosclerosis, and thrombotic diseases, Adv. Gerontol., 2013, vol. 3, no. 4, pp. 243254.

Kuznik, B.I., Khavinson, V.Kh., Tarnovskaya, S.I., et al., Epigenetic effect of Lys-Glu and Ala-Glu-Asp-Gly peptides on the hemostasis system, cytokine profile, and life span, Usp. Klin. Med., 2014, nos. 1-2, pp. 29-42.

Kuznik, B.I., Khavinson, V.Kh., Linkova, N.S., et al., Growth factors of fibroblasts FGF19, FGF21, FGF23 as endocrine regulators of physiological functions and geroprotectors: epigenetic regulatory mechanisms, $U_{s p}$. Sovrem. Biol., 2017, vol. 137, no. 1, pp. 84-99.

Lin'kova, N.S., Drobintseva, A.O., Orlova, O.A., et al., Peptide regulation of skin fibroblast functions during their aging in vitro, Bull. Exp. Biol. Med., 2016, vol. 161, no. 1 , pp. 175-178.

Morozov, V.G. and Khavinson, V.Kh., Isolation, purification, and identification of an immunomodulatory polypeptide found in the thymus of calves and humans, Biokhimiya (Moscow), 1981, vol. 46, no. 9, pp. 1652-1659.

Morozov, V.G., Khavinson, V.Kh., Kozhemyakin, A.L., and Kozhemyakin, L.A., Influence of the polypeptide factor of thymus on the cyclic nucleotide system of immunocompetent cells, Vopr. Med. Khim., 1982, vol. 28, no. 4 , pp. 114-118. 
Morozov, V.G., Khavinson, V.Kh., and Malinin, V.V., Peptidnye timomimetiki (Peptide Thymomimetics), St. Petersburg: Nauka, 2000.

Ryzhak, A.P., Chalisova, N.I., Linkova, N.S., et al., The effect of polypeptides on cell regeneration in the culture of different tissues of young and old rats, Usp. Gerontol., 2015, vol. 28, no. 1, pp. 97-103.

Ryzhak, A.P., Chalisova, N.I., Lin'kova, N.S., et al., Effect of polypeptides on cell proliferation and apoptosis during aging, Bull. Exp. Biol. Med., 2017, vol. 162, no. 4, pp. 534-538.

Sevostianova, N.N., Linkova, N.S., Polyakova, V.O., et al., Immunomodulating effects of Vilon and its analogue in the culture of human and animal thymus cells, Bull. Exp. Biol. Med., 2013, vol. 154, no. 4, pp. 562-565.

Shcherbak, V.A. and Pateyuk, A.V., The effect of Vilon on the immune response in acute immobilization stress in rats, Sib. Med. Zh., 2004, vol. 44, no. 3, pp. 26-29.

Smirnov, V.S., Petlenko, S.V., and El'tsin, S.S., The use of thymogen for preoperative preparation of elderly patients with tumor in the abdominal cavity, Usp. Gerontol., 2011, vol. 24, no. 2, pp. 278-284.

Tang, N., Bai, H., Chen, X., et al., Anticoagulant treatment is associated with decreased mortality in severe coronavirus disease 2019 patients with coagulopathy, $J$.
Thromb. Haemostasis, 2020, vol. 18, pp. 1094-1099. https://doi.org/10.1111/jth.14817

Terekhov, A.Yu., Kormilets, D.Yu., Linkova, N.S., et al., Peptide KE in human proteome, Bull. Exp. Biol. Med., 2020, vol. 168, no. 5, pp. 631-633.

Wang, D., Hu, B., Hu, C., et al., Clinical characteristics of 138 hospitalized patients with 2019 novel coronavirusinfected pneumonia in Wuhan, China, J. Am. Med. Assoc., 2020, vol. 323, pp. 1061-1069.

https://doi.org/10.1001/jama.2020.1585

Zakutskii, A.N., Chalisova, N.I., Aniskina, A.I., et al., The tissue-specific effect of synthetic peptides in organotypic tissue cultures in young and old rats, Usp. Gerontol., 2006, no. 19, pp. 93-96.

Zhang, C., Wu, Z., Li, J.-W., et al., Cytokine release syndrome in severe COVID-19: interleukin-6 receptor antagonist tocilizumab may be the key to reduce mortality mortality, Int. J. Antimicrob. Agents, 2020, vol. 55, art. ID 105954.

https://doi.org/10.1016/j.ijantimicag.2020.105954

Zhurkovich, I.K., Kovrov, N.G., Ryzhak, G.A., et al., Identification of short peptides as part of polypeptide complexes isolated from animal organs, Usp. Sovrem. Biol., 2020, vol. 140, no. 2, pp. 140-148. 